\title{
Spectral content of Pc5-6/Pi3 geomagnetic pulsations and their efficiency in generation of geomagnetically induced currents
}

\author{
Ya. A. Sakharov ${ }^{1,2}$, N. V. Yagova ${ }^{2,3}$, V. A. Pilipenko ${ }^{2,3}$, and V. N. Selivanov ${ }^{4}$
}

Received 20 September 2021; accepted 24 November 2021; published 18 February 2022.

ULF geomagnetic pulsations till recent time have been an underestimated factor of space weather hazard to technological systems. The efficiency of GIC generation by geomagnetic pulsations depends on frequency and spatial distribution of the pulsation's magnetic field. To clarify how the spectral content of Pc5-6/Pi3 (periods 3-15 min) geomagnetic pulsations influences their efficiency in GIC generation, the inter-relations between amplitudes of GIC and magnetic field variations are studied statistically. For that, we use the data of four-year (2014-2018) continuous GIC recording at the Vykhodnoy auroral station and geomagnetic pulsations at the nearest stations of IMAGE magnetometer network. Potentially risky $10 \mathrm{~A}$ GICs can originate from non-storm pulsations with amplitudes about few tens of nanotesla which are common at auroral latitudes. On average, multi-harmonic pulsations are more effective in GIC generation that 1-harmonic ones. The pulsations producing essential GICs preferably occur at pre-noon hours under moderate auroral activity. KEYWORDS: Geomagnetic pulsations; geomagnetically induced currents; power transmission lines.

Citation: Sakharov, Ya. A., N. V. Yagova, V. A. Pilipenko, and V. N. Selivanov (2022), Spectral content of Pc5-6/Pi3 geomagnetic pulsations and their efficiency in generation of geomagnetically induced currents, Russ. J. Earth. Sci., 22, ES1002, doi:10.2205/2021ES000785.

\section{Introduction}

GICs in electric power lines (EPLs) are generated during geomagnetic disturbances in a contour formed by the EPL, grounding, and the Earth's crust. GICs cause damage of electric equipment because of processes in the transformers and incorrect functioning of protecting systems, and in extreme cases even blackouts [Boteler, 2019, Pilipenko, 2021. The most intensive GICs were

\footnotetext{
${ }^{1}$ Polar Geophysical Institute, Apatity, Murmansk region, Russia

${ }^{2}$ Geophysical Center of the RAS, Moscow, Russia

${ }^{3}$ Schmidt Institute of Physics of the Earth of the RAS, Moscow, Russia

${ }^{4}$ Northern Energetics Research Center, Kola Science Center of the RAS, Kola, Murmansk region, Russia
}

Copyright 2022 by the Geophysical Center RAS. http://rjes.wdcb.ru/doi/2021ES000785-res.html observed during main phase of violent magnetic storms (e.g. [Kappenman, 2005; Pulkkinen et al., 2006 Trichtchenko et al., 2007]).

ULF geomagnetic pulsations in the $\mathrm{Pc} 5-6 / \mathrm{Pi} 3$ band (periods 3-15 min) till recent time have been an underestimated factor of GIC-associated hazard to technological systems. However, their long duration (several hours on average), large amplitudes (sometimes comparable with weak substorm), and $d B / d t$ absolute values, which showed their efficiency in GIC estimates [Viljanen, 1997], make to consider ULF activity as a serious threat to power lines [Kataoka and Pulkkinen, 2008]. GICs associated with high amplitude ULF waves in the $\mathrm{Pc5} / \mathrm{Pi} 3$ frequency range were as high as those registered during substorm onsets [Apatenkov et al., 2020. Heyns et al., 2021. Mostly the GIC proxies $-d B / d t$ or electrotelluric fields are estimated from magnetic field records along with models of the Earth conductivity (e.g. [Love et al., 2017] and 


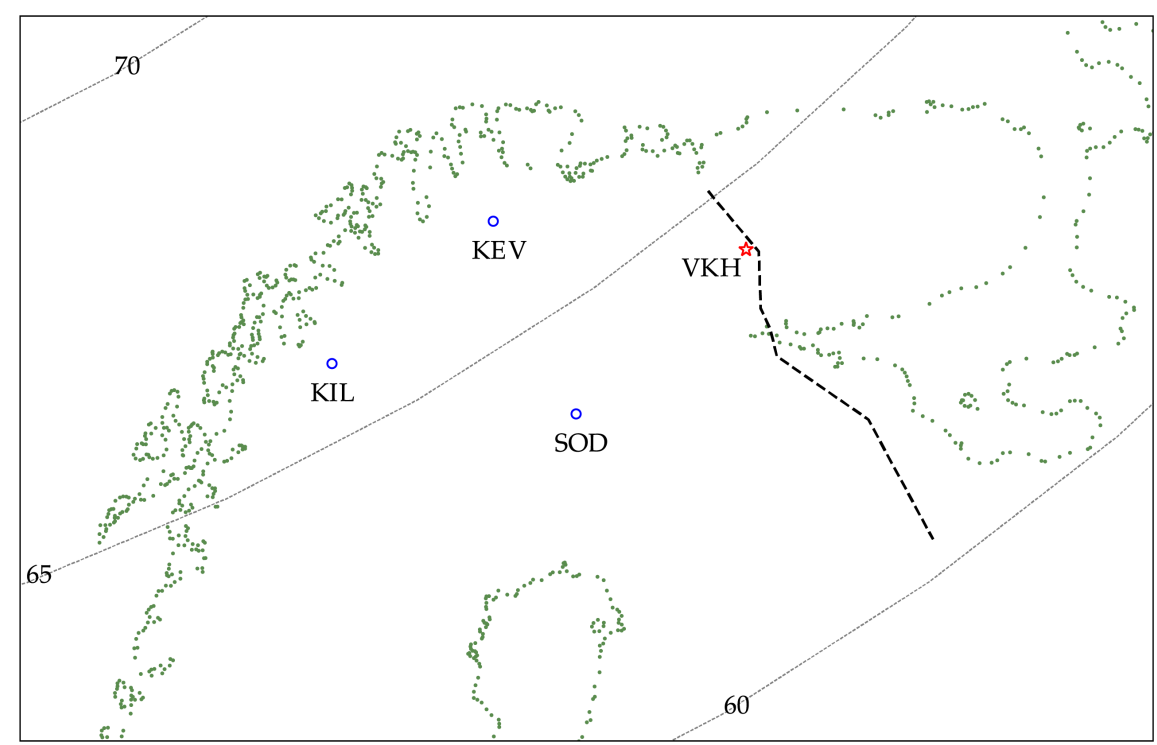

Figure 1. A scheme of magnetic and GIC measurements. Magnetic stations are shown with blue circles, the EPL "Northern Transit" is shown with a dashed line, and VKH station where GIC is measured is shown with a star. Grey lines indicate CGM parallels.

references therein). Studies based on direct GIC measurements [Belakhovsky et al., 2019, Heyns et al., 2021, Kozyreva et al., 2020] are not so numerous. Both approaches have advantages and disadvantages. Magnetotelluric modelling allows to accumulate information from the entire area around power transmission line under question. However, only direct measurements of GICs can reveal factors which have not been included into a model yet. Contribution of these parameters can be estimated from a statistical analysis of simultaneously measured magnetic field and GIC variations. The studies in which model results are validated with the direct measurements (e.g. [Blake et al., 2018, Dimmock et al., 2019, Wik et al., 2008]) combine the advantages of both approaches but are the most time-consuming.

The parameters which influence efficiency of a geomagnetic disturbance in GIC generation are not identical for magnetic storms and geomagnetic pulsations. As disturbances caused by a magnetic storm are global, GIC intensity is controlled by geomagnetic field time variations, as well as by spatial distribution of the conductivity of the Earth's crust and the parameters of electric circuit (see e.g. [Arajärvi et al., 2011, Boteler and Pirjola, 2017 and references therein). Spatial scale of geomagnetic pulsations also influences GIC amplitudes. In our previous study [ Yagova et al., 2021], the dependence of spatial scale of Pc5/Pi3 geomagnetic pul- sations on their efficiency in GIC generation was revealed. GIC to magnetic field amplitude ratio can also depend on spectral content of geomagnetic pulsations. If at least two spectral maxima exist in the pulsation spectra, the coincidence of harmonic phase can lead to enhanced level of $d B / d t$ and thus GIC amplitudes.

In the present study we examine a possible influence of the ULF spectral content on the efficiency of GIC generation by Pc5-6/Pi3 geomagnetic pulsations using data of several years of GIC and geomagnetic observations in the Russian North-West and Fennoscandia.

\section{Observational Data and Their Processing}

GICs were recorded with 1-min cadence at the terminal station Vykhodnoj (VKH) of the "Northern Transit" power transmission line [Sakharov et al., 2009. The geomagnetic field data were taken from the nearest 3 stations of IMAGE magnetometer network [Tanskanen, 2009]. Stations record three components of geomagnetic field with $10 \mathrm{~s}$ cadence. A map showing the locations of magnetic stations, GIC-recording site, and electric power line is presented in Figure 1. 
Table 1. Station Information

\begin{tabular}{|c|c|c|c|c|c|c|}
\hline \multirow[t]{2}{*}{$\begin{array}{l}\text { Station } \\
\text { code }\end{array}$} & \multirow[t]{2}{*}{$\begin{array}{l}\text { Measurement } \\
\text { code }\end{array}$} & \multicolumn{2}{|c|}{ Geographic } & \multicolumn{2}{|c|}{$\begin{array}{c}\text { Corrected } \\
\text { geomagnetic (CGM) }\end{array}$} & \multirow[t]{2}{*}{$\begin{array}{c}\mathrm{UT} \text { at } \\
\mathrm{MLT}=0\end{array}$} \\
\hline & & Latitude & Longitude & Latitude & Longitude & \\
\hline KIL & $B$ & 69.02 & 20.79 & 66.13 & 102.80 & $21: 28$ \\
\hline $\mathrm{KEV}$ & $B$ & 69.76 & 27.01 & 66.65 & 108.35 & 21:06 \\
\hline SOD & $B$ & 67.37 & 26.63 & 64.22 & 106.52 & $21: 13$ \\
\hline $\mathrm{KH}$ & $I$ & 68.83 & 33.08 & 65.53 & 112.73 & $20: 49$ \\
\hline
\end{tabular}

The same stations have been used in [Yagova et al., 2021 to separate ULF disturbances into largescale and small-scale pulsations. Station information is summarized in Table 1. Type of measurements is codified as $B$ and $I$ for magnetic field and GIC measurements, respectively. The last column gives universal time (UT) of magnetic local midnight. The data for 4 years from July 2014 to June 2018 is used for the analysis. These years cover the maximum and at the declining phase of the 24-th solar cycle. During this period the intervals of fast solar wind were common and thus Pc5/Pi3 amplitudes are expected to be elevated.

\subsection{Data Processing}

The "Northern Transit" electric power line is elongated along the meridian. Therefore, we use for the analysis $B_{Y}$ (eastward) component because it corresponds better to GIC variations as compared with $B_{X}$ (northward) component [Sakharov et al., 2021. The frequency band under consideration $1.0-5.5 \mathrm{mHz}$ comprises narrow-band Pc5 waves and broadband Pi3/Ps6 pulsations. We do not intentionally discriminate quasi-monochromatic (Pctype) and irregular (Pi-type) pulsations. Our analysis is based on criteria which quantify pulsation spectra and its possible influence on GIC generation.

The raw magnetometer data have been preliminary low-pass filtered with a cutoff frequency $8.3 \mathrm{mHz}$ and decimated to the 1-minute time step. Prior to spectral analysis, the magnetic field 1-min data have been high-pass filtered with the cut-off frequency $f_{c}=0.8 \mathrm{mHz}$. Further we use the notations $b_{Y}$ and $\Delta I$ for variations of $B_{Y}$ component and GIC to discriminate them from their undisturbed values. Cross-spectra are estimated with the Blackmann-Tukey method [Jenkins and Watts, 1969 Kay, 1988 in the 64 points (3840 s) time window. Power-spectral density (PSD), spectral coherence $\gamma^{2}$ and phase difference $\Delta \phi$ have been applied for the classification of pulsations. Spectral coherence and phase difference are calculated for two stations to estimate spatial scale of pulsations. The correspondence between GICs and geomagnetic pulsations is estimated from data at VKH and KEV stations. As a measure of ULF power at VKH meridian, the PSD along the profile KEV-KIL has been linearly extrapolated to this location.

To suppress the influence of spatial scale of geomagnetic pulsations on their efficiency in GIC generation, only large-scale pulsations are taken for the analysis. To discriminate between large-scale and small-scale pulsations, we use spectral coherence $\gamma^{2}$, PSD ratio $R$, and phase difference $\Delta \phi$ from in the North-South $(\mathrm{N}-\mathrm{S})$ and East-West $(\mathrm{E}-\mathrm{W})$ directions in the same technique as in [Yagova et al., 2021. Numerical values of the parameters at which pulsations are classified as large-scale are summarized in Table 2 .

We attribute ULF disturbances to GIC-associated pulsations if $I-b_{Y}$ coherence is as high as $\gamma_{I B}^{2}>$ 0.5 , and peak-to-peak amplitudes of GIC and $b_{Y}$ variations exceed $4 \mathrm{~A}$ and $8 \mathrm{nT}$, respectively. For both $b_{Y}$ and GIC pulsations we introduce square mean amplitude $A_{Y}$ according to

Table 2. Values of the Parameters to Classify Pc5/Pi3s as Large-Scale Pulsations

\begin{tabular}{|c|c|c|c|}
\hline Parameters & $\gamma^{2}$ & $R$ & $\mu=\cos (\Delta \phi)$ \\
\hline$\overline{\mathrm{N}-\mathrm{S}}$ & $>0.7$ & $0.5-2$ & $>0.75$ \\
\hline $\mathrm{E}-\mathrm{W}$ & $>0.7$ & $0.67-1.33$ & Any \\
\hline
\end{tabular}




$$
A_{Y}^{2}=\sum_{n=1}^{N} Y_{n}^{2} / N
$$

where $Y=(b$ or $I)$, and $N$ is number of points in the time interval. To quantify efficiency of geomagnetic pulsations in GIC generation, a parameter $R_{I B}$ is introduced as $R_{I B}=A_{I} / A_{b}$.

In order to study possible influence of pulsations' spectral shape on their GIC efficiency, we divide all the large-scale pulsations into groups in accordance with their spectral content, and parameters of GIC $-b_{Y}$ inter-relation. Group 1 includes all the largescale $b_{Y}$ pulsations with over-threshold amplitudes. Group 2 is a part of group 1 and includes pulsations producing over-threshold GIC pulsations with $\gamma_{I B}>0.5$. Actually, this group includes the pulsations for which GIC has an over-threshold amplitude and it is likely generated by the geomagnetic pulsations. However, even within this group, GIC amplitudes differ essentially for the same $b_{Y}$ amplitudes. Group 3 includes group 2 pulsations with over-threshold values of $R_{I B}>0.2 \mathrm{~A} / \mathrm{nT}$. Pulsations of this group are more effective in GIC generation in comparison with the others.

Pulsations of all the three groups are divided into 1-harmonic and multi-harmonic ones according to the follows spectral criteria. A pulsation is considered 1-harmonic if its $d b_{Y} / d t$ spectra contains a major maximum with halfwidth which does not exceed a threshold value $W_{b}=1 \mathrm{mHz}$ at a given height $h_{b}=0.3 \mathrm{PSD}_{\max }$, and it is either the only spectral maximum, or PSD ratio of major to each of minor maxima $R_{\mathrm{MAJ}}$ exceeds the threshold value $\left(R_{\mathrm{MAJ}}>3.75\right)$. If a spectrum has two or more maxima with $R_{\mathrm{MAJ}}$ below the threshold value, a pulsation is considered a multi-harmonic one. The condition of narrow spectral width eliminates from consideration step-like variations of geomagnetic field (e.g. during substorm onset) and isolated impulses [Engebretson et al., 2020.

A relative time duration of each group occurrence as compared with total duration of observational period $\left(3.5 \times 10^{4}\right.$ hours $)$ is given in Table 3. For all the pulsation groups, multi-harmonic pulsations are more frequent in comparison with 1-harmonic ones.
Table 3. Percentage of Total Duration of Each Group of Geomagnetic Pulsations to the Duration of the Whole 4-Year Interval of the Analysis

\begin{tabular}{lcc} 
Group $\backslash$ Pulsations & 1-harmonic & Multi-harmonic \\
\hline 1 & $1.3 \%$ & $5.3 \%$ \\
2 & $0.05 \%$ & $0.2 \%$ \\
3 & $0.026 \%$ & $0.12 \%$ \\
\hline
\end{tabular}

\section{Examples}

Different efficiency of geomagnetic pulsations in GIC generation is illustrated with the following examples. Figure 2 shows the ULF event recorded near the magnetic midnight on 16 August (day 228) 2015 at the early recovery phase of a moderate magnetic storm with minimal $D s t=-84 \mathrm{nT}$ started on the previous day. The pulsation has a visible period about 11 minutes which corresponds to $1.3 \mathrm{mHz}$ frequency of PSD maxima for both of geomagnetic and GIC pulsations. The geomagnetic pulsations have peak-to-peak amplitude of $b_{Y}$ component about $100 \mathrm{nT}$ and maximal $d b_{Y} / d t$ variations about $0.5 \mathrm{nT} / \mathrm{s}$. Maxi-

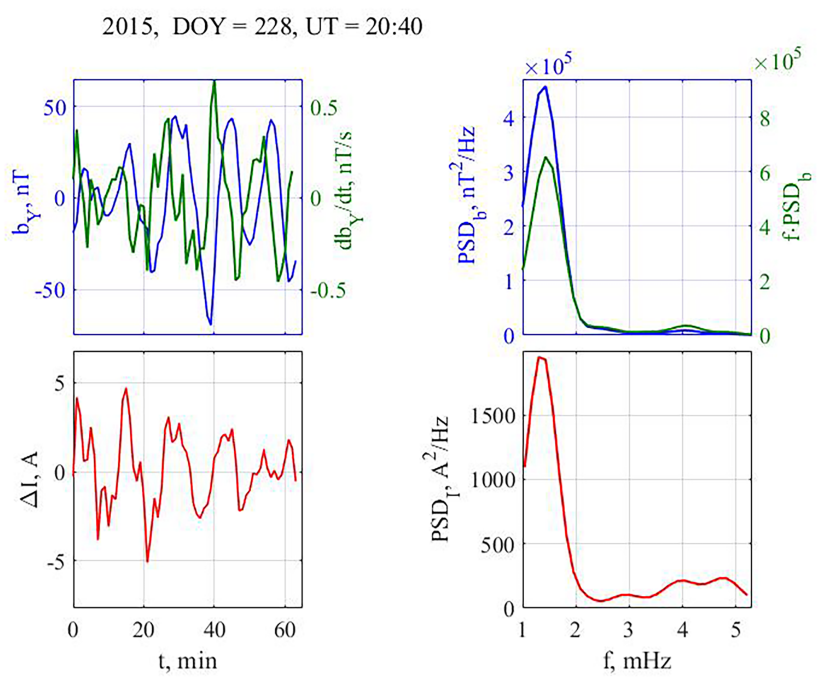

Figure 2. An example of geomagnetic (top) and GIC (bottom) pulsations with one dominating spectral maximum. Pulsation waveforms are shown in left-hand panels and their PSD are shown in right-hand panels. The $b_{Y}$ and $d b_{Y} / d t$ waveforms and spectra are shown in blue and green, respectively, and the scales for them are given in left-hand/right-hand axes. 

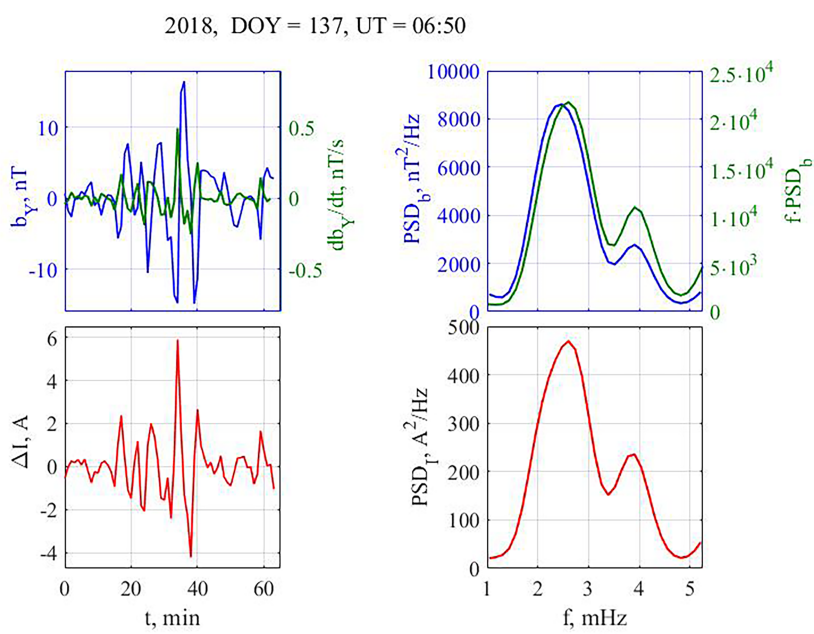

Figure 3. An example of geomagnetic and GIC pulsations with two spectral maxima. The format of the Figure is the same as in Figure 2.

mal peak-to-peak amplitude of resulting GIC variations is about $10 \mathrm{~A}$. The GIC generation efficiency $R_{I B} \approx 6 \times 10^{-2} \mathrm{~A} / \mathrm{nT}$.

However, GIC pulsations with comparable amplitudes can result from much weaker geomagnetic disturbances. An example of non-storm geomagnetic pulsations and resulting GIC variations is presented in Figure 3.

The pulsation event was recorded on 17 May (day 137) 2018 (MLT is about 10 at VKH meridian). Maximal peak-to-peak amplitudes of geomagnetic and GIC pulsations are about $25 \mathrm{nT}$ and $10 \mathrm{~A}$, respectively. Waveforms of geomagnetic pulsations has several fragments with $d B_{Y} / d t \sim 0.5 \mathrm{nT} / \mathrm{s}$, i.e. with approximately the same magnitude as for pulsations with $100 \mathrm{nT}$ amplitude shown in Figure 2. Their GIC generation efficiency is several times higher than in previous event, $R_{I B} \sim 0.25 \mathrm{~A} / \mathrm{nT}$. This is a result of the interference of two harmonics in PSD spectra with frequency ratio about $3 / 2$ at 2.7 and $4 \mathrm{mHz}$.

\section{Statistics}

In this section efficiency $\left(R_{I B}\right)$ of 1-harmonic and multi-harmonic Pc5/Pi3 pulsations in GIC generation is compared for the whole 4-year interval. Figure 4 presents $R_{I B}$ empirical probability density functions (PDF) for 1-harmonic and multiharmonic pulsations.

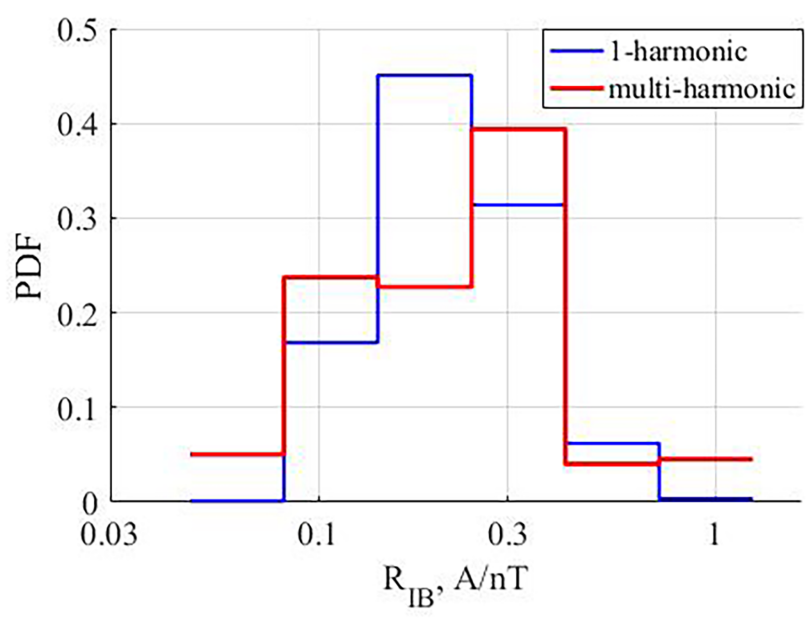

Figure 4. Distribution of 1-harmonic and multi-harmonic pulsations over $R_{I B}$ amplitude ratios for all the $\mathrm{Pc} 5 / \mathrm{Pi} 3$ intervals selected in 2014-2018.

The Figure 4 shows that the most probable value of $R_{I B}$ is nearly 1.5 times higher for the multiharmonic pulsations than for those with the only frequency dominating.

The parameters of geomagnetic pulsations cannot be completely predicted from the extra-magnetospheric factors (e.g. [Alperovich and Fedorov, 2007). However, it is well known that Pc5 pulsations have specific diurnal variation with a major maximum in the morning MLT sector and a minor one at afternoon hours [Baker et al., 2003, while Pi3 pulsations are typical at night hours. Diurnal variation of the GIC intensity has a maximum during the nighttime hours, caused by substorms, and a maximum at early morning hours, associated with Pi3/Pc5 activity [Vorobev et al., 2019].

Diurnal variation (in MLT) for 3 groups Pc56/Pi3 pulsations is illustrated in Figure 5 separately for 1-harmonic multi-harmonic pulsations. It is seen from the Figure 5 that the main maximum in the occurrence of $\mathrm{Pc} 5 / \mathrm{Pi} 3$ s producing GICs (groups 2 and 3) is found at pre-noon hours for both 1-harmonic and multi-harmonic pulsations. Meanwhile, diurnal variations of all the large-scale pulsations (group 1) differ in dependence of the spectral content. For them, the main maximum is shifted towards noon for multi-harmonic pulsations, as compared to 1-harmonic ones. Minor post-noon maximum is also seen in both multi-harmonic and GIC-effective 1-harmonic pul- 
sations (group 3). Diurnal variations of 1-harmonic pulsations (groups 2 and 3 ) also demonstrate maxima in the pre-midnight sector, probably associated with Pi3 pulsations. Summarizing, we can conclude that morning and pre-noon hours are favorable for Pc5/Pi3 geomagnetic pulsations producing GICs.

Broadband Pi3 and Ps6 pulsations are associated with auroral activations [Kleimenova et al., 2002]. At auroral latitudes pulsation activity is controlled mostly by auroral activations measured by the auroral electrojet $(A E)$ index, or a similar non-official SME (SuperMag Electrojet) index [Gjerloev, 2009]. The SME index PDFs for the same groups of pulsations as in Figure 5, are presented in Figure 6.

It is seen from the Figure 6 that the distributions for GIC producing pulsations (groups 2 and 3) are shifted to higher SME values as compared with all the large-scale pulsations. The maxima are found at $\mathrm{SME} \geq 300 \mathrm{nT}$, against $150 \mathrm{nT}$ for all the largescale pulsations. These distributions also indicate the difference between 1-harmonic and multiharmonic pulsations. From the present analysis we can conclude Pc5/Pi3 geomagnetic pulsations producing GICs are more probable under moderate auroral activity resulting in SME index values exceeding $300 \mathrm{nT}$. Meanwhile the difference in SME distributions between pulsations with different $R_{I B}$ values is not essential.
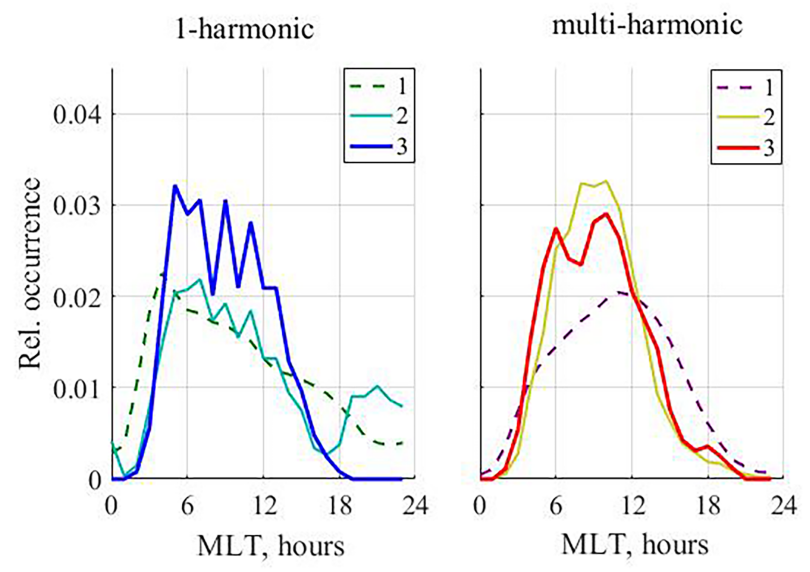

Figure 5. Diurnal variation for 3 groups of largescale 1-harmonic (left) and multi-harmonic (right) Pc5/Pi3s. Group numbers are indicated at legends.

\section{Discussion}

The most important result of the above analysis is that GIC with comparable amplitudes can be generated by both high-amplitude storm-time Pc5-6/Pi3 pulsations and by non-storm ones with moderate amplitudes. The $R_{I B}$ distribution shown in Figure 4 shows that maximal $R_{I B}$ values are about 1 . This means that the $\mathrm{Pc} 5-6 / \mathrm{Pi} 3$ pulsations with typical amplitudes about few tens nT cause of potentially risky GICs variations with amplitudes about few tens A, while rare extreme GICs with amplitudes about of higher than 100 A originate from pulsations with extremely high amplitudes at the main or recovery phase of a magnetic storm [Apatenkov et al., 2020, Wik et al., 2008].

The large-scale $\mathrm{Pc5}-6 / \mathrm{Pi} 3$ pulsations producing GICs of detectable amplitudes are most probable at moderate auroral activity at morning and prenoon hours. Multi-harmonic pulsations are more effective in GIC generation in comparison with 1-harmonic ones. A higher efficiency of multiharmonic pulsations follows from the existence of a second harmonic which has a higher frequency (for a uniform conductivity of the Earth crust the components of amplitude spectra are related as $I(f)=$ $\left.f^{1 / 2} B(f)\right)$. However, this is not the only reason for the difference in GIC efficiency of 1-harmonic and multi-harmonic pulsations. Figure 7 shows frequency PDFs of number of events and energy for both 1-harmonic and multi-harmonic pulsations.

Rather unexpectedly, the PDF is shifted towards high frequencies for 1-harmonic pulsations
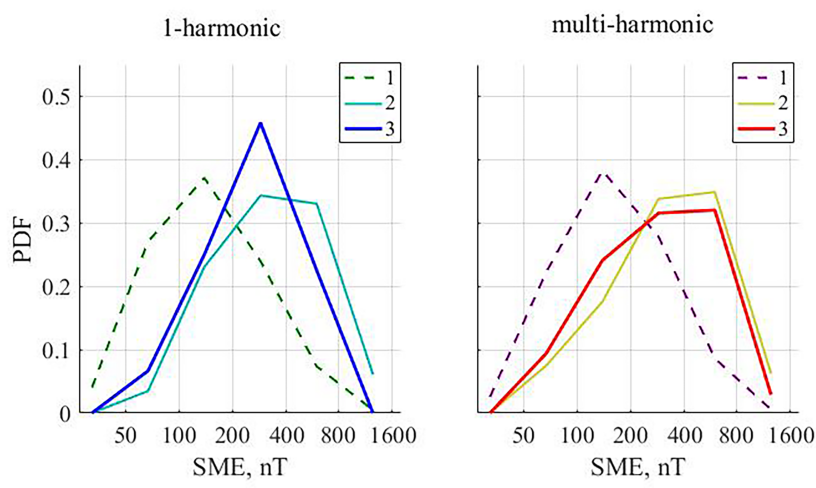

Figure 6. Distribution over SME index for 3 groups of large-scale $\mathrm{Pc} 5 / \mathrm{Pi} 3$ pulsations. Their group numbers are indicated in legends. The format is the same as in Figure 5. 

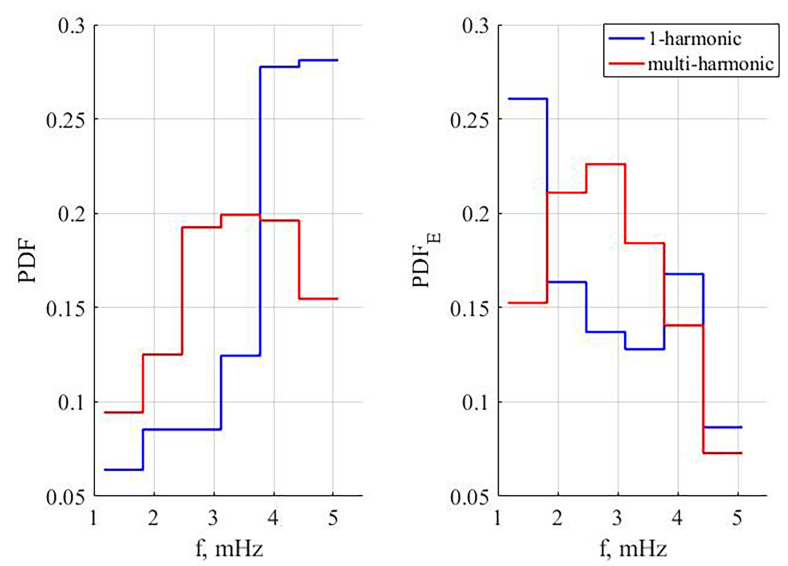

Figure 7. Frequency distributions for number of events $\left(\mathrm{PDF}\right.$, left) and energy $\left(\mathrm{PDF}_{E}\right.$, right) for 1-harmonic and multi-harmonic pulsations.

as compared with multi-harmonic ones. While PDF of multi-harmonic pulsations has a wide maximum centered at $3.5 \mathrm{mHz}$, the PDF maximum for 1-harmonic pulsations is found above $4 \mathrm{mHz}$. The $\mathrm{PDF}_{E}$ is shifted to lower frequencies in comparison with the PDF for both groups of pulsations. This is due to the fact that averaged PSD decreases with frequency. For 1-harmonic pulsations, energy distribution has two maxima centered at 1.5 and 4 $\mathrm{mHz}$. They can be associated with Pi3 and Pc5 pulsations. As for the latter frequency band, it coincides with the one of Alfven FLR at the magnetic latitudes studied. The details of FLR behavior of azimuthal $\left(b_{Y}\right)$ component are studied in [Lifshicz and Fedorov, 1986, and this effect probably causes non-monotonous frequency dependence of GIC to $b_{Y}$ PSD ratio for some groups of pulsations, found in [Yagova et al., 2021]. This allows to assume, why 1-harmonic pulsations are less effective GIC generation than multi-harmonic ones even under the same $d b_{Y} / d t$ values obtained from the measurements at a short base along a meridian. GICs are controlled by distribution of pulsation magnetic field over the whole EPL length. Meanwhile, magnetic field of resonant pulsations are strongly nonmonotonous along a meridian. However, the pulsations can be classified as large-scale if the points used for the selection are not co-located with region of high amplitude and phase gradients. Besides, in the present study, the nearest magnetometer is displaced at 4.5 degrees westward from the EPL meridian. This leads to additional requirements on
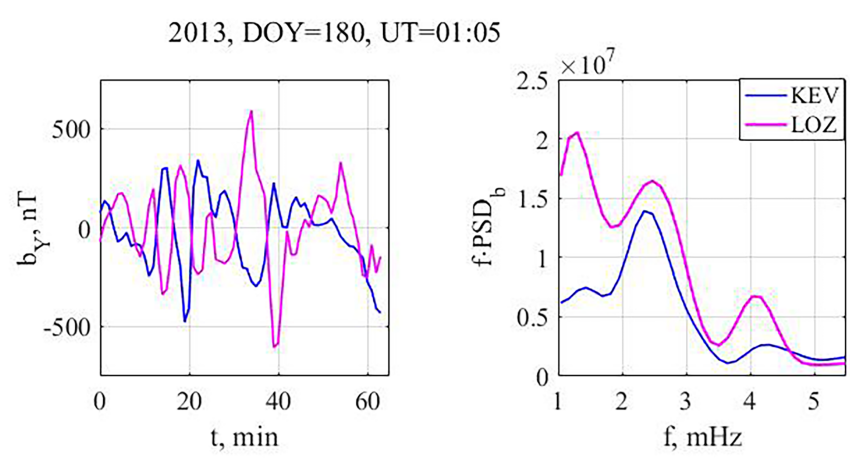

Figure 8. Waveforms (left) and $d b_{Y} / d t$ PSD spectra (right) for the storm-time Ps6 pulsation described in [Apatenkov et al., 2020.

pulsations' amplitude and phase gradients in the $\mathrm{E}-\mathrm{W}$ direction and can lead to additional inaccuracy in $R_{I B}$ estimates.

In contrast to expectations, statistical diurnal variations have shown that substantial number of Pc5 pulsations belong to multi-harmonic events. The occurrence of higher harmonics may be associated with distortions of the wave forms which make them non-sinusoidal and with steep fronts which leads to increase of GIC amplitudes.

Actually, GICs associated with geomagnetic pulsations are comparable with those caused by auroral substorm onsets. However, they can be studied quantitatively with an accuracy sufficient for applications only under optimal configuration of magnetic measurements. The distances between the EPL and magnetic stations, and between adjacent stations are limited from above by a scale of variation of pulsation's amplitude and phase and from below by the interference produced by EPL. Meanwhile, existing networks of magnetic stations are too rare for GIC applications, especially for the EPLs prolongated along a meridian in which GICs originate predominantly from pulsation $b_{Y}$ component. Meanwhile, neglected $b_{X}$ component may contribute to GIC owing to anisotropy of the crust and isotropy of small-scale structure embedded into the auroral geomagnetic activity.

During the event with extreme values of the peak-to-peak GIC in the "Northern Transit" line $I \sim 200 \mathrm{~A}$ and $d B / d t \sim 6.5 \mathrm{nT} / \mathrm{s}$ GIC bursts were caused by quasi-periodic sequence of magnetic pulses (Ps6 pulsations) with amplitudes $b \sim$ $500-1000 \mathrm{nT}$ [Apatenkov et al., 2020, Chinkin et al., 2021]. The pulsation efficiency in this event is 
$R \sim 200 / 1000 \sim 0.2 \mathrm{~A} / \mathrm{nT}$, that is about the same as in the example in Figure 3.

Waveforms and $d b_{Y} / d t$ spectra for these pulsations are shown in Figure 8 for the KEV station used as a base one in the present study and at LOZ located closer to the EPL meridian $\left(\Phi=64.2^{\circ}\right.$, $\left.\Lambda=114.2^{\circ}\right)$. LOZ was not used in the present study because its data coverage during the years of analysis is not enough for statistics. The spectra are calculated for a 64 minutes interval starting at 01:05 UT in the technique used in the present research. The pulsation demonstrates complex waveforms and multi-harmonic spectra at both stations. The steepest fragments of $b_{Y}$ variations with time are found at LOZ, as well as higher PSD and contribution of minor spectral peaks to total energy. This pulsation illustrates that even storm-time disturbances which are usually large-scale may have essential gradients along a latitude. Both waveforms and spectra demonstrate non-negligible difference between pulsations at two stations separated by $6^{\circ}$ in longitude. This can result in additional inaccuracy in GIC applications. In fact, an analysis based on magnetic and GIC measurements separated by several degrees would either produce essential errors in extrapolation of geomagnetic pulsations' parameters to the EPL meridian or take into account a very limited number of large-scale events. Actually, the low fraction of large-scale events summarized in Table 3 can be essentially increased if only a meridional magnetometer chain located within 12 degrees from the "Northern Transit" EPL meridian existed and its data could be utilized along with the data recorded by IMAGE.

\section{Conclusion}

At auroral latitudes, non-storm Pc5-6/Pi3 pulsations with moderate amplitudes are an important source of potentially risky GICs with amplitudes up to few tens $\mathrm{A}$. The diurnal variations and dependence on auroral activity of all the largescale Pc5-6/Pi3 pulsations and ones associated with GICs are not identical. The maximal occurrence of Pc5-6/Pi3 pulsations associated with GICs is found at pre-noon hours under moderate (SME 200 - 600 nT) auroral activity.

The spectral content of ULF pulsations is found to be a significant factor of the GIC generation efficiency. Multi-harmonic pulsations are more effective in GIC generation in comparison with 1-harmonic ones. The most probable value of $R_{I B}$ is nearly 1.5 higher for multi-harmonic pulsations than for one harmonic-ones.

Acknowledgments. We are grateful to the institutions that maintain the IMAGE network. We gratefully acknowledge the SuperMAG collaborators (https://sup ermag.jhuapl.edu/info/?page=acknowledgement). The study (Ya.S., N.Y., V.P.) is supported by grant \# 2177-30010 from the Russian Science Foundation.

\section{References}

Alperovich, L. S., E. N. Fedorov (2007), Hydromagnetic waves in the magnetosphere and the ionosphere, Astrophysics and Space Science Library, vol. 353 p. 418, Springer, Netherlands. Crossref

Apatenkov, S. V., V. A. Pilipenko, et al. (2020), Auroral omega bands are a significant cause of large geomagnetically induced currents, Geophysical Research Letters, 47, e2019GL086677, Crossref

Arajärvi, E., R. Pirjola, A. Viljanen (2011), Effects of neutral point reactors and series capacitors on geomagnetically induced currents in a high-voltage electric power transmission system, Space Weather, 9, S11005, Crossref

Baker, G., E. F. Donovan, B. J. Jackel (2003), A comprehensive survey of auroral latitude Pc5 pulsation characteristics, J. Geophys. Res., 108, No. 1384, A10, Crossref

Belakhovsky, V., V. Pilipenko, et al. (2019), Impulsive disturbances of the geomagnetic field as a cause of induced currents of electric power lines, $J$. Space Weather and Space Climate, 9, A18, Crossref

Blake, S. P., P. T. Gallagher, et al. (2018), A detailed model of the Irish high voltage power network for simulating GICs, Space Weather, 16, 1770-1783, Crossref

Boteler, D. H. (2019), A 21st century view of the March 1989 magnetic storm, Space Weather, 17, 1427-1441, Crossref

Boteler, D. H., R. J. Pirjola (2017), Modeling geomagnetically induced currents, Space Weather, 15, 258-276, Crossref

Chinkin, V. E., A. A. Soloviev, V. A. Pilipenko, et al. (2021), Determination of vortex current structure in the high-latitude ionosphere with associated GIC bursts from ground magnetic data, J. Atm. SolarTerr. Phys., 212, 105514, Crossref

Dimmock, A. P., L. Rosenqvist, et al. (2019), The GIC and geomagnetic response over Fennoscandia to the 7-8 September 2017 geomagnetic storm, Space Weather, 17, 989-1010, Crossref 
Engebretson, M. J., K. R. Kirkevold, et al. (2020), Interhemispheric comparisons of large nighttime magnetic perturbation events relevant to GICs, J. Geophys. Res.: Space Physics, 125, e2020JA028128, Crossref

Gjerloev, J. W. (2009), A Global Ground-Based Magnetometer Initiative, EOS, 90, 230-231, Crossref

Heyns, M. J., S. I. Lotz, C. T. Gaunt (2021), Geomagnetic pulsations driving geomagnetically induced currents, Space Weather, 19, e2020SW002557, Crossref

Jenkins, G. M., D. G. Watts (1969), Spectral Analysis and its Application, 525 pp. Holden day, San Francisco, London, Amsterdam.

Kappenman, J. G. (2005), An overview of the impulsive geomagnetic field disturbances and power grid impacts associated with the violent Sun-Earth connection events of 29-31 October 2003 and a comparative evaluation with other contemporary storms, Space Weather, 3, S08C01, Crossref

Kataoka, R., A. Pulkkinen (2008), Geomagnetically induced currents during intense storms driven by coronal mass ejections and corotating interacting regions, J. Geophys. Res., 113, A03S12, Crossref

Kay, S. M. (1988), Modern Spectral Estimation: Theory and Application, 543 pp. Prentice-Hall, New Jersey.

Kleimenova, N. G., O. V. Kozyreva, et al. (2002), Case studies on the dynamics of $\mathrm{Pi} 3$ geomagnetic and riometer pulsations during auroral activations, Ann. Geophys., 20, 151-159, Crossref

Kozyreva, O., V. Pilipenko, et al. (2020), Fine structure of substorm and geomagnetically induced currents, Annals of Geophysics, 63, No. 2, GM219, Crossref

Lifshicz, A. E., E. N. Fedorov (1986), Hydromagnetic oscillations of the magnetosphere-ionosphere reson tor, Docl. USSR Acad. Sci., 287, 90-95.

Love, J. J., P. A. Bedrosian, A. Schultz (2017), Down to Earth With an Electric Hazard From Space, Space Weather, 15, 658-662, Crossref

Pilipenko, V. A. (2021), Space weather impact on ground-based technological system, Solar-Terrestrial Physics, 7, No. 2, 3-12, Crossref
Pulkkinen, A., A. Klimas, et al. (2006), Spatiotemporal scaling properties of the ground geomagnetic field variations, J. Geophys. Res., 111, No. A3, A03305, Crossref

Sakharov, Ya. A., A. N. Danilin, et al. (2009), Geomagnetically induced currents in the power systems of the Kola peninsula at solar minimum, Proc. of 8th Symp. on Electromagnetic Compatibility and Electromagnetic Ecology p. 237-238, St. Petersburg Electrotechnical University, St. Petersburg.

Sakharov, Ya. A., N. V. Yagova, V. A. Pilipenko (2021), Pc5/Pi3 geomagnetic pulsations and geomagnetically induced currents, Bull. Russ. Acad. Sci. Phys., 85, 329-333, Crossref

Tanskanen, E. I. (2009), A comprehensive highthroughput analysis of substorms observed by IMAGE magnetometer network: Years 1993-2003 examined, J. Geophys. Res., 114, A05204, Crossref

Trichtchenko, L., A. Zhukov, et al. (2007), November 2004 space weather events: Real-time observations and forecasts, Space Weather, 5, S06001, Crossref

Viljanen, A. (1997), The relation between geomagnetic variations and their time derivatives and implications for estimation of induction risks, GRL, 24, 631-634, Crossref

Vorobev, A. V., V. A. Pilipenko, et al.

(2019), Statistical relationships between variations of the geomagnetic field, auroral electrojet and geomagnetically induced currents, Solar-Terrestrial Physics, 5, No. 1, 35-42, Crossref

Wik, M., A. Viljanen, et al. (2008), Calculation of geomagnetically induced currents in the $400 \mathrm{kV}$ power grid in southern Sweden, Space Weather, 6, S07005, Crossref

Yagova, N. V., V. A. Pilipenko, et al. Spatial scale of geomagnetic $\mathrm{Pc} 5 / \mathrm{Pi} 3$ pulsations as a- a factor of their efficiency in generation of geomagnetically induced currents, Earth, Planets and Space, Crossref

Corresponding author:

N. V. Yagova, Geophysical Center of the Geophysical Center of the Russian Academy of Science, 3 Molodezhnaya St., 119296, Moscow, Russia. (nyagova@yandex.ru) 